\title{
Research and Simulation of High Speed Artillery Projectile Cluster Target Resolution
}

\author{
Song CHunji \\ Ordnance Engineering College of PLA \\ Shijiazhuang, China \\ 15200024516@163.com \\ * Corresponding Author \\ Shi Lin \\ Ordnance Engineering College of PLA \\ Shijiazhuang,China \\ 15200024516@163.com
}

\author{
Han ZHuangzhi \\ Ordnance Engineering College of PLA \\ Shijiazhuang,China \\ 15200024516@163.com
}

\begin{abstract}
Due to high firing rate artillery projectile firing rate is high, many targets exist in the beam at the same time, the cluster target resolution for projectile muzzle velocity measuring is important. The three-dimensional resolution technique based on time-distance-frequency is an effective method to solve the problem of high speed target resolution. In this paper, the three-dimensional resolution based on pseudo code ranging is applied, a simulation of cluster target resolution using Simulink is made, analyzes and verifies the advanced method based on the threedimensional resolution, and puts forward two methods to reduce the distance gate leakage, provides a reference for project demonstration and engineering implementation of time-distance-frequency three-dimensional resolution of velocity measurement radar.
\end{abstract}

Keywords-Cluster Target Resolution; Three-Dimensional Resolution Based on Time-Distance-Frequency; Advanced and Correctness; Pseudo Code Ranging; Distance Gate Leakage

\section{INTRODUCTION}

With the high speed projectile firing rate increasing, cluster resolution for projectile velocity measurement is essential. Cluster resolution technology has been a research hotspot in the field of radar signal processing. Cluster resolution can be considered from the distance, angle, speed difference between the target differences, can also be used in combination to obtain better resolution performance difference [1]. Aiming at the resolution problem of high speed projectile target, to distinguish cluster according to three-dimensional resolution based on range resolution, through the launch of a continuous wave signal by pseudo random code phase modulation, the effective distance of the beam is divided into a plurality of distance interval and achieve multi objective space division [2]. Analyze frequency of each target distance interval, to further improve the resolving effect of group target.

Simulink is a software package for modeling, simulation and analysis of dynamic systems. It provides a graphical interactive environment [3]. This paper uses the Simulink to build a cluster resolution simulation system, it is divided into a plurality of target echo signal processing module, a distance gate correlation processing module, a decimation filter module and a band-pass filter module four parts. Through the simulation, this paper verifies the correctness of the three-dimensional resolution technique based on time-distance-frequency. M series with a single code length pseudo code modulation waveforms can effectively cluster target range resolution, but the side lobes cause the energy leakage, a great impact the target resolution seriously. We propose a method to reduce the spectrum leakage problem caused by the pseudo code modulation and demodulation based on the engineering practice, it provides a reference for the three dimensional resolution scheme and the engineering realization of the velocity measurement radar.

In the second part of this paper the method of high speed projectile group target resolution is analyzed. Part 3 for the high speed projectile cluster resolution system simulation and analysis results are given. The fourth part summarizes.

\section{Method of High SpeEd Projectile GrouP TARGET RESOLUTION}

At present, there are two main types of methods for multi target resolution by low resolution radar: one is time frequency analysis method through Doppler domain [4-5]. The defect of this method is that it only uses the Doppler information, and does not have the large ability to distinguish. The second is a feature extraction method based on low resolution radar echo [6-7]. This kind of method uses the useful echo features, combined with time spectrum information (the aircraft track, the speed and the target movement characteristics), to realize aircraft type, size and number identification. But the neural network or template matching method is necessary for the final recognition, It is obviously not feasible to train the various types, various vehicles or to build templates. And this method needs more related information, is not conducive to rapid resolution. Compared with the two dimensional resolution based on distance-Doppler from literature [8], and it has higher resolution through three-dimensional resolution technique based on time-distance-frequency in this paper.

Taking into account the order of the projectile goes successively through the chamber. The projectile core is 
arranged in the order in the radar beam. In different time, different distance, there are targets with different speed (Doppler frequency), as shown in Figure 1. In the picture, the horizontal coordinate is the distance, the vertical axis is the time and the middle is the Doppler frequency.

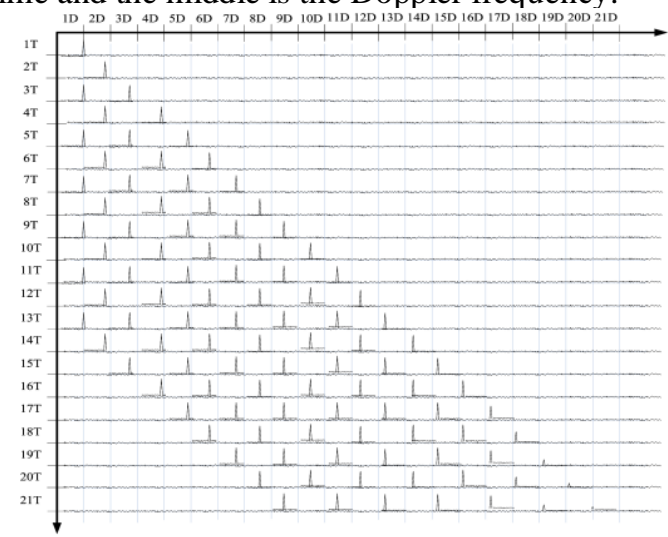

Figure 1. The Target of the Projectile in the Time - Distance - Doppler Multi Dimensional Distribution

"Target one" in the first period of time in the first range, in the second period of time in the second range, the rate has dropped. In the third period, the "target two" appears in the first range, while the "target one" appears in the third range, the speed continues to decline, the magnitude has dropped. In the fourth period, the target one, target two were flying to second, fourth range, the speed range has been reduced, until the subsequent emergence of target three, target four, etc. Through the analysis, it can achieve the group target resolution very well rely on a proper resolution of time and distance [2].

Continuous wave radar is used to measure the velocity, but it is necessary to modulate the transmitted signal in order to realize the ranging function. Commonly used modulation method has linear frequency modulation, pseudo random code phase modulation. Pseudo code phase modulation continuous wave radar has big time bandwidth product, has a good range and velocity resolution, and therefore the choice of pseudo random code phase modulation system. Emission waveforms are shown in Figure 2.

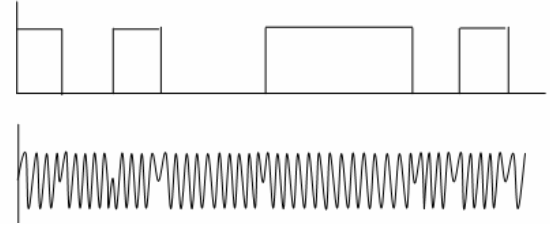

Figure 2. Pseudo Random Code and Modulated Emission Waveform

The $\mathrm{m}$ sequence is a typical pseudo random sequence, which has the characteristic of sharp auto correlation with Gauss white noise [9-10]. M sequence auto correlation function:

$$
R(\tau)=\left\{\begin{array}{cccc}
1 & \tau=0 & \bmod & p \\
-\frac{1}{p} & \tau \neq 0 & \bmod & p
\end{array}\right.
$$

The autocorrelation function of the $m$ sequence is shown in Figure 3.

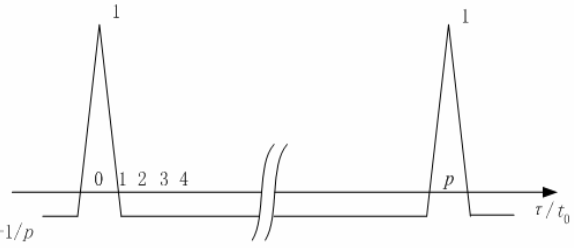

Figure 3. Auto Correlation Curve of $\mathrm{M}$

In the picture, $\Delta$ is pseudo-random code width, $\mathrm{T}$ is pseudo-random code repeat cycle, and $\mathrm{P}$ is pseudo-random code length. The frequency of the code clock determines the distance resolution, the code clock and length determine the repetition period of the pseudo random encoding, and the no fuzzy distance of the radar.

Using the autocorrelation of $m$ sequence, the copy of the transmitted signal is used to carry out the relevant calculations with the echo mixed with noise, and then determine the target distance by measuring the position of the maximum value of the correlation function. The simulation system in the distance gate correlated processing module produces PN code signal after a certain time delay, the time delay " $\tau$ " corresponding to the radial distance $r$ is the distance corresponding to the distance gate value [11]

$$
R=\frac{c^{*} \tau}{2}
$$

\section{High SPEed PRojectile Cluster Resolution SIMULATION}

The whole system is shown in Figure 4, which can be divided into four parts, which are the target echo generation module, the distance gate correlation processing module, the extraction filter module and the band pass filter module.

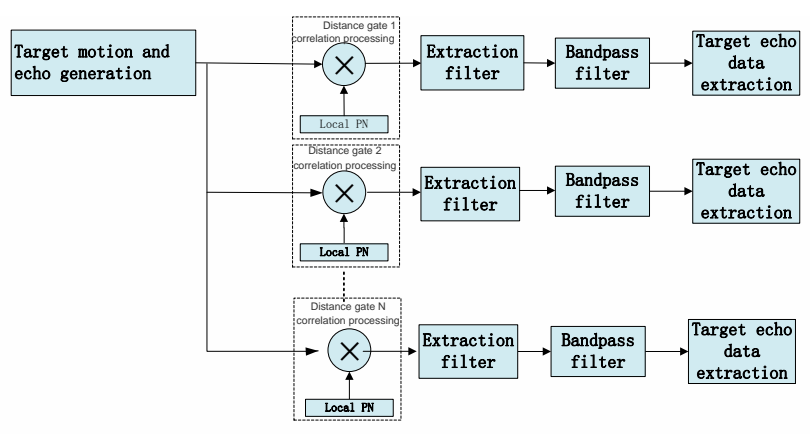

Figure 4. Composition of Simulation System

\section{A. Signal Processing Module}

The echo baseband signal makes correlation demodulation with local pseudo random code after shift in the different distance gate. If the echo signal is a certain time delay, the Doppler signal is obtained by the corresponding distance channel after correlation and filtering. The distance and speed information of the target can be obtained by the FFT processing and target detection, to provide the basis for the resolution of the group target. The signal processing flow is shown in Figure 5.

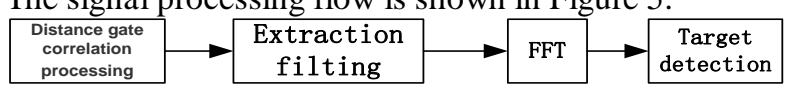

Figure 5. Signal Processing Flow Chart 


\section{B. Simulation Process}

Setting goals for eleven tube 30mm APDS. The main parameters for simulation: projectile firing interval about $6 \mathrm{~ms}$, projectile muzzle velocity $1200 \mathrm{~m} / \mathrm{s}$, acceleration $150 \mathrm{~m} / \mathrm{s} 2$, card the same valve velocity and projectile and acceleration about for $-1000 \mathrm{~m} / \mathrm{s} 2$, carrier frequency $33.6 \mathrm{KMHz}$, PN code clock $25 \mathrm{MHz}$, pseudo code length 255 , sampling rate of $300 \mathrm{MHz}$.

1) Single objective situation: If there is only one core target in space, the position of the radar and the target is shown in Figure 6.

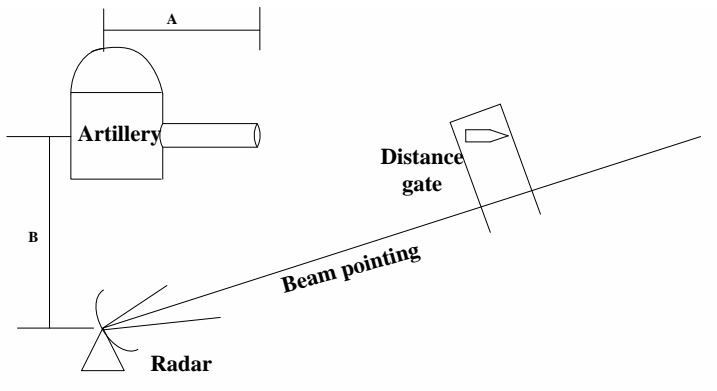

Figure 6. Single Target and Radar Position Diagram

The echo signal waveform processed by a distance gate correlation processing is shown in Figure 7. If the radial distance of the target is within the range of correlation processing, the signal waveform is shown in Figure 7 (a), on the contrary, as shown in Figure 7 (b).

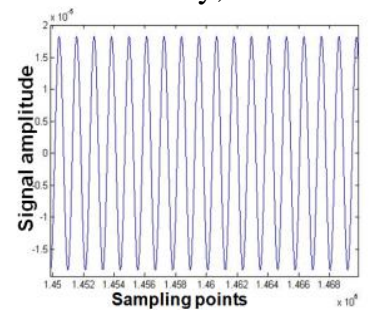

(a)

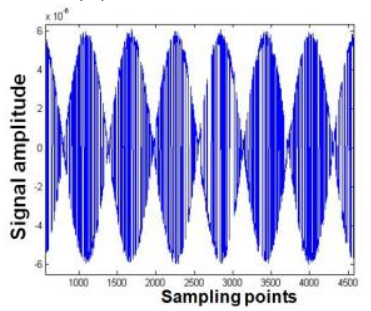

(b)
Figure 7. Signal Waveform after Correlation Processing

The signal waveform shown in Figure 7 (a) after FFT processing is shown in Figure 8 (a), which can be seen as a result of the spectral analysis of the ideal Doppler single frequency signal. The signal waveform shown in Figure 7 (b) after FFT processing is shown in Figure 8 (b), which can be seen as the spectrum of Doppler signal, which is the result of the previous pseudo random code spectrum after translation.

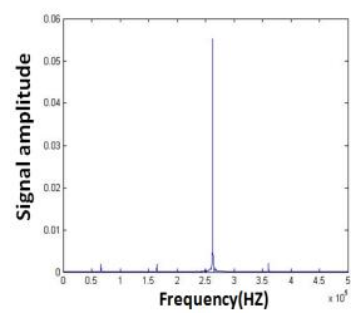

(a)

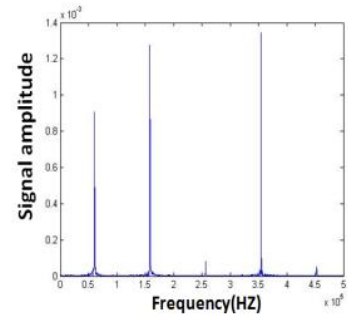

(b)
Figure 8. FFT Signal Waveform

The above analysis shows that the distance and time frequency resolution method based on the pseudo code modulation can be performed to distinguish the distance from different targets in the range of distance dimension parallel correlation processing. In the speed dimension, the targets which the distance is close, but the speed is different are distinguished by the FFT processing or the time-frequency processing, to achieve good resolution.

But the results shown in Fig. 8 can be analyzed, the echo signal spectrum lines after correlation processing, which the targets are outside the gate, influence the targets inside. Spectrum leakage is mainly generated by the periodic of $\mathrm{m}$ sequence pseudo-random code. There are two kinds of discrete spectral lines in the band, one is the "pseudo random code zero spectrum line" of target Doppler frequency. Two is the "pseudo random code periodic spectrum line" which is interval with "pseudo random code zero spectrum line " \pm fcode/, \pm 2 fcode/N... Spectral line leakage as shown in Figure 9.

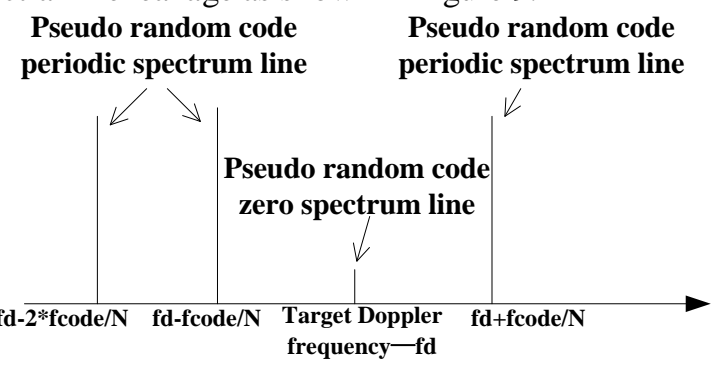

Figure 9. Leakage Schematic Diagram

The spectral line energy of the leakage is related to the distance and the code length. The position is related to code clock, the code length and the target Doppler. Spectral range of the "pseudo random code zero spectrum line" caused by the targets (Set radial distance dis1) outside the gate is about the targets (Set radial distance dis2) inside dis22/ $\left(\mathrm{N}^{*}\right.$ dis12). Spectral range of the " pseudo random code periodic spectrum line" caused by the targets (Set radial distance) outside the gate is about the targets (Set radial distance) inside dis $2^{2 /}\left(\sqrt{\mathbb{N}} * \operatorname{dis} 1^{2}\right)$.

2) Multi objective situation: There are three core targets in space, and the core target corresponds to a card target. Radar, target location diagram shown in Figure 10.

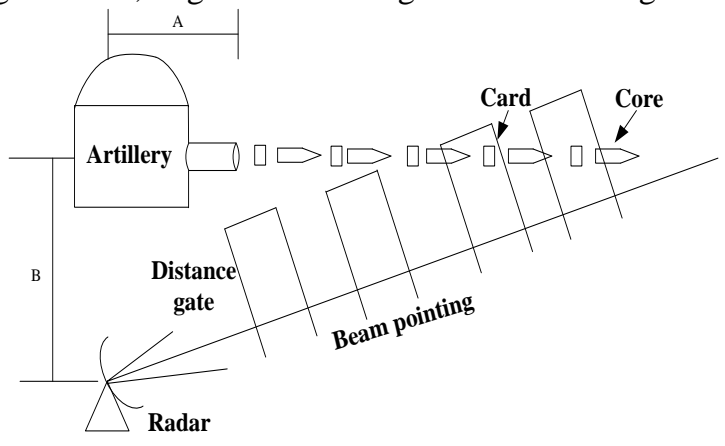

Figure 10. The Location Relationship between High Firing Rate

Weapon and Radar

Figure 11 shows the signal spectrum before and after band pass filter, the signal spectrum is generated by the 3 way distance gate correlation processing. 3 way distance gate is [204m 210m], [402m 408m], [756m 762m], the left side is before the filtering, the right side is after. 

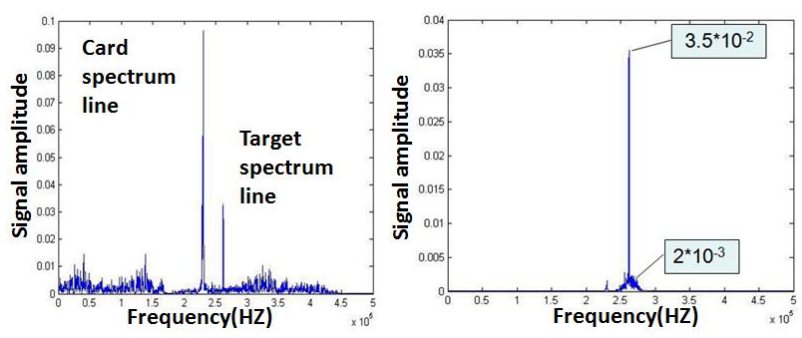

(a) $[204 \mathrm{~m} 210 \mathrm{~m}]$
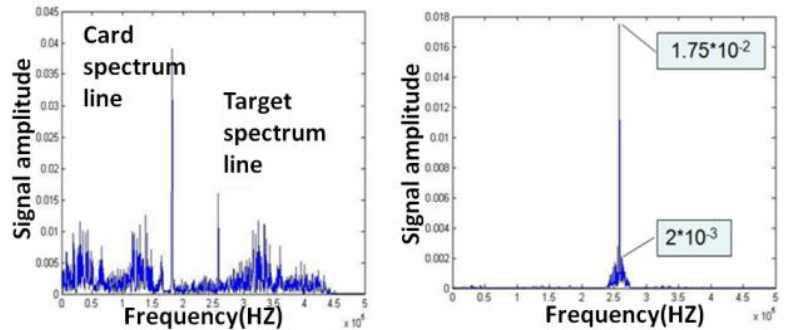

(b) $[402 \mathrm{~m} \mathrm{408m]}$
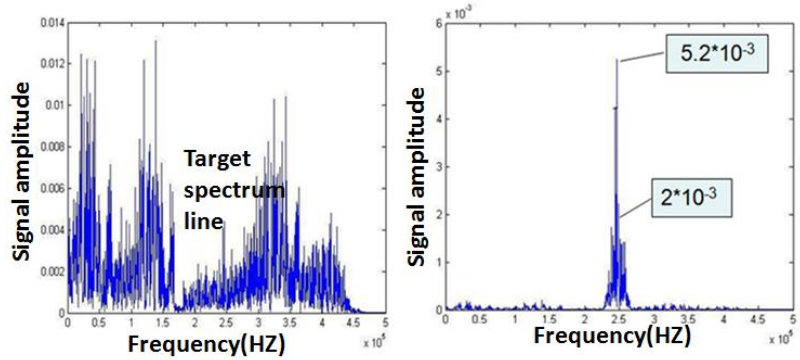

(c) $[756 \mathrm{~m} 762 \mathrm{~m}]$

Figure 11.Spectrum of Different Distance Gate with Bandpass Filter

According to Figure 11, we can conclude that the energy of the target spectrum in the range of the distance is decreased with the increase of the radial distance (Target signal range $3.5 * 10-2 \sim 5.2 * 10-3)$, but the "leakage" caused by the core and the card outside the distance is basically the same (Target signal range $3.5 * 10-2 \sim 5.2 * 10-3$ ). The reason is that the amplitude of the side lobe of the auto correlation function of $\mathrm{m}$ sequence is constant. In the distance gate $[756 \mathrm{~m} 762 \mathrm{~m}]$, it can be analyzed that the "pseudo random code periodic spectrum line" of the close range of the projectile and the card valve has affected the detection of the target spectrum. Therefore, it is very important to reduce the influence of the near distance target for the distant target.

In summary, the most influence of the detection is the close to the radar. There are two ways reducing the "leakage": First is to adjust the antenna's point, so that the antenna side lobe whose gain is lower points to these goals.
Second is to choose a better band pass filter, to filter out the "pseudo random code periodic spectrum line" which is far away from the target spectrum line.

\section{CONCLUSIONS}

Aiming at high speed artillery projectile cluster target resolution, we study the three-dimensional resolution based on time-distance-frequency, analyze the ranging principle of $\mathrm{m}$ sequence pseudo-random code. The process of group target resolution is simulated by Simulink, and the simulation results are analyzed. We propose two methods to reduce the distance gate side lobe leakage problem in pseudo code modulation and demodulation system. Practice has proved that it can effectively reduce the impact of leakage. When the actual project implementation process, it can adjust the related parameters according to the analysis of this paper, so as to effectively achieve high speed artillery projectile cluster target resolution.

\section{REFERENCES}

[1] ZHAI Qinglin,CHEN Fubin and ZHANG Jun, "A Review of Modern Radar Signal Processing Methods for Group Target Detection," DIANGUANG YU KONGZHI, vol.14, Dec.2007, pp. 95-98.

[2] ZHANG Hongwei, JU Feng and HE Qiang, "Study on measurement technology of high speed gun muzzle velocity bursts," DANJIAN YU ZHIDAO XUEBAO,vol.34,Feb.2014,pp. 176-179.

[3] YAO Jun, MA Songhui, "Simulink modeling and simulation," Xi'an Electronic and Science University press, 2002.

[4] SUN Xiaobing, BAO ZHeng and LUO Lin, "Time frequency signal analysis and multi object resolution of radar," XITONGGONGCHENG YU DIANZIJISHU, Jun.1997,pp. 12- 16.

[5] LI Yong, LIU Yuping and LIAN Baowang, "Application of weighted rearrangement algorithm in multi target radar signal resolution," DIANZI YU XINXI XUEBAO, Jan.2003,pp. 31- 36.

[6] SONG Yingfeng, ZHANG Hanhua and JIANG Weidong, "Study on feature extraction and classification method of low resolution radar target echo waveform," LEIDA YU DUIKANG, Dec.2002,pp. 1- 5.

[7] WANG Wei, ZHANG Hanhua and JIANG Weidong, "Target feature extraction method for low resolution radar," GUOFANGKEJIDAXUE XUEBAO,Apr.2002,pp. 31- 35.

[8] DU Lan,LIU Hongwei and BAO ZHeng, "A group target resolution method based on distance Doppler," DIANZI XUEBAO,Jun.2004,pp. 81-85.

[9] FAN Meimei , "Improved digital receiving system of the individual reconnaissance radar," Chinese people's Liberation Army Ordnance Engineering College,2007.

[10] PAN Minghai,CHEN ZHenghua and WEI ZHongmin, "Super resolution detection of beam target of the missile borne pulse Doppler radar antenna," NANJING HANGKONGHANGTIANDAXUE XUEBAO,Apr.2008,pp. 220225.

[11] LIN Kexiang, WANG Yifei, "The principle and application of pseudo random code," People's Posts and Telecommunications Press, 1978. 